'Instituto de Matemática y Física, Universidad de Talca. Talca, Chile. 2Departamento de Salud Pública, Facultad de Ciencias de la Salud, Universidad de Talca. Talca, Chile. ${ }^{3}$ Escuela de Medicina, Facultad de Ciencias de la Salud, Universidad de Talca. Talca, Chile. ${ }^{4}$ Violence Prevention Research Program, Department of Emergency Medicine, UC Davis School of Medicine. Sacramento, CA, USA.

aEstadística, PhD Biometrics. bOdontóloga, MS Public Health. Internos/a Medicina, Universidad de Talca.

${ }^{d}$ Antropólogo, Doctor Salud Pública.

Financiación: Álvaro CastilloCarniglia recibe financiación de Becas Chile-CONICYT y del Robertson Fellow in violence prevention research.

Los autores no declaran conflictos de interés.

Recibido el 23 de junio de 2017 , aceptado el 24 de octubre de

Correspondencia a: Gloria Icaza Dirección postal: Casilla 721, Código postal 3460000, Talca, Chile.

Teléfono oficina: 56712200338 gicaza@utalca.cl

\section{Asociación entre variables demográficas y socioeconómicas y mortalidad por cirrosis y otras enfermedades del hígado}

\author{
GLORIA ICAZA ${ }^{1, a}$, LORETO NÚÑEZ2,b, \\ NICOLÁS ORDAZ R. ${ }^{3, \mathrm{c}}$, CONSTANZA VERDUGO W., ${ }^{3, \mathrm{c}}$, \\ SERGIO CAGLIERI S. ${ }^{3, \mathrm{c}}$, ÁLVARO CASTILLO-CARNIGLIA ${ }^{4, \mathrm{~d}}$
}

\begin{abstract}
Background: Cirrhosis is a serious public health problem worldwide. There are geographical, socioeconomic and demographic differences in mortality due to the disease. Aim: To establish an association between mortality from cirrhosis and other chronic liver diseases and socioeconomic and demographic indicators in communes of the two largest regions of Chile, the Metropolitan Region (RM) and Biobio. Material and Methods: Analysis of the mortality data from the Chilean Ministry of Health. Multiple regression models of smoothed standardized mortality ratios at the community level between 2001 and 2008, were carried out for men and women in relation to socioeconomic and demographic indicators. Results: Quite dissimilar phenomena were observed in these two regions. In RM, the risk of death is associated with urban communes of lower educational level $\left(R^{2}=53.6 \%\right.$ in men, $R^{2}=62.3 \%$ in women $)$. In men of the Biobio Region, the risk decreases along with the percentage of population belonging to originary populations $\left(R^{2}=9.1 \%\right)$. In women, the model also includes a variable that represents the service sector $\left(R^{2}=15.0 \%\right)$, that represents a greater risk. Conclusions: The association of mortality due to liver disease with other variables, changes according to the territory in which it is studied. Therefore, specific local studies are required to address this problem in depth. These studies will contribute to the design of locally relevant public policies, aimed at addressing health inequities and the prevention of liver diseases.
\end{abstract}

(Rev Med Chile 2017; 145: 1412-1420)

Key words: Demography; Liver Cirrhosis; Mortality; Socioeconomic Factors.
L a exposición a diversos agentes como virus, toxinas y drogas determina alteraciones del hígado a nivel estructural y funcional, las cuales generan, en etapas avanzadas, la insuficiencia crónica del órgano y su correlato anatomopatológico más frecuente, la cirrosis hepática ${ }^{1}$.

Se estima a nivel mundial una tasa de morta- lidad por cirrosis al año 2010 de 15,8 por 100.000 personas-año ${ }^{2}$. En ese mismo año se registraron en Chile 4.285 defunciones $(4,4 \%$ del total de muertes) por cirrosis y otras enfermedades crónicas del hígado, con una tasa de mortalidad específica de 25,1 por cada 100.000 habitantes; esto la ubica como tercera causa de muerte luego 
de enfermedades cerebrovasculares $(52,0 / 100.000$ habitantes) y enfermedades isquémicas del corazón (45,6/100.000 habitantes) $)^{3}$. Cabe hacer notar que el número de muertes debido a cirrosis y otras enfermedades crónicas del hígado en hombres es 2,4 veces el de mujeres. Entre los años 1990 y 2007 se ha evidenciado en Chile una disminución de las tasas de mortalidad por cirrosis alcohólica y no alcohólica ${ }^{4}$ concordante a lo observado en estudios internacionales ${ }^{2}$. En países occidentales aproximadamente $90 \%$ de los casos de cirrosis hepática pueden atribuirse a tres causas: abuso de alcohol, enfermedad por hígado graso no alcohólica y hepatitis crónica vírica ${ }^{5}$.

La relación entre ingesta excesiva de alcohol y aumento en la prevalencia y mortalidad por cirrosis hepática se encuentra consistentemente descrita en la literatura ${ }^{6}$. La asociación entre consumo de alcohol y cirrosis es tan estrecha, que se emplea a la mortalidad por cirrosis hepática como indicador de la tasa de problemas graves relacionados con el alcohol ${ }^{7}$.

En Chile, $48,9 \%$ de la población entre 12 y 64 años declara consumo reciente de alcohol, de los cuales $43,7 \%$ presenta una ingesta abusiva o síntomas de dependencia ${ }^{8}$. De acuerdo al Estudio de Carga de Enfermedad y Carga Atribuible, el año 2007 el consumo problemático de alcohol fue responsable de la pérdida de $12,4 \%$ del total de años de vida saludables (AVISA), ubicándose así en primer lugar entre los factores de riesgo evitables 9 . Se estima que $21,5 \%$ de los años de vida potenciales perdidos (AVPP) serían consecuencia de la ingesta de alcohol. La cirrosis hepática sería la principal responsable de las muertes atribuidas al consumo de alcohol a nivel nacional, por sobre el suicidio y los accidentes de tránsito ${ }^{10}$. Se han identificado y caracterizado extensamente el rol de los indicadores de nivel socioeconómico y demográfico en sus distintas dimensiones (educación, recursos financieros, ocupación, etnicidad) sobre las diferencias observadas en la mortalidad general y específica de diferentes poblaciones ${ }^{11}$. En Chile se observa poca literatura al respecto, a pesar de la importancia de esta patología en la carga de enfermedad 9 .

Hasta el año 2016, Chile se dividía administrativamente en 15 regiones, de las cuales la Región Metropolitana y la Región del Bíobío presentan el mayor riesgo de mortalidad por cirrosis, identificándose en ambas conglomerados de comunas con exceso de riesgo ${ }^{12}$. Fenómeno que no se observa en otras regiones.

El objetivo de este estudio fue evaluar la asociación a nivel comunal entre diversos indicadores socioeconómicos y demográficos y el riesgo de mortalidad por cirrosis y otras enfermedades del hígado en las regiones Metropolitana y del Bíobío.

\section{Métodos}

Se realizó un estudio ecológico para explorar la relación entre el riesgo de mortalidad por cirrosis y otras enfermedades crónicas del hígado (códigos K70, K72.1, K73, K74, K76.0.1.9 de la $10^{a}$ revisión de la Clasificación Internacional de Enfermedades [CIE-10]) con variables socioeconómicas y demográficas en las comunas de las regiones Metropolitana $(n=52)$ y del Bíobío $(\mathrm{n}=54)$. Las comunas (346 a nivel nacional) son la unidad político-administrativa más pequeña en Chile. Este trabajo se circunscribe en las regiones Metropolitana y del Bíobío que son las más pobladas, representando en su conjunto $52,4 \%$ de la población nacional según último censo 2002. La motivación para esta elección se relaciona con que ambas regiones presentan elevado riesgo de mortalidad por cirrosis con respecto al resto del país en hombres y mujeres, con numerosas comunas en alto riesgo ${ }^{12}$. Junto con esto, la Región del Bíobío presenta el mayor porcentaje de comunas con elevado riesgo de mortalidad atribuible al alcohol comparado con el resto del país ${ }^{13}$.

El riesgo de mortalidad desagregado a nivel comunal se obtuvo a partir de la base de datos derivada de la publicación Atlas de Mortalidad en Chile 2001-2008 ${ }^{12}$, realizada a partir de los datos de mortalidad del Departamento de Estadísticas e Información en Salud (DEIS) del Ministerio de Salud $^{3}$. El riesgo de mortalidad, variable respuesta, se expresó en términos de la Razón de Mortalidad Estandarizada suavizada (RMEs) ${ }^{12}$, que se calculó en dos etapas, primero se estandarizaron las tasas de mortalidad por el método indirecto, del período 2001-2008, que es la suma de los casos observados en los ocho años dividido por los casos esperados en el mismo período, resultando en la Razón de Mortalidad Estandarizada. Esta razón se sometió a un método de suavizamiento mediante modelos de regresión Poisson bayesianos ${ }^{14}$. Se consideró alto riesgo valores de RMEs 30\% mayor a lo esperado y riesgo bajo a valores 30\% menor 
a lo esperado. El detalle metodológico del suavizamiento bayesiano se encuentra disponible en Icaza, y otros, $2013^{12}$.

Para caracterizar el nivel socioeconómico y demográfico de las comunas, variables explicativas, se seleccionaron indicadores de: educación, ingresos, ocupación, etnicidad y ruralidad cuya información estuviera comprendida dentro del período de estudio, obtenidos del estudio "Diagnósticos Regionales con enfoque de Determinantes Sociales de la Salud"15:

1) Los indicadores de educación e ingresos fueron elaborados con datos de la Encuesta de Caracterización Socioeconómica Nacional (CASEN) del año $2006^{16}$, según la metodología utilizada por el Índice de Desarrollo Humano (IDH) ${ }^{17}$.

2) El porcentaje de población rural y el porcentaje de población perteneciente a pueblos originarios, fueron obtenidos de la CASEN $2006^{16}$.

3) El porcentaje de población inserta en los distintos sectores productivos fueron obtenidos a partir de datos del Instituto Nacional de Estadísticas (INE) a través del Sistema Nacional de Información Municipal para el año 2002. Estos indicadores se clasificaron como primario, secundario y terciario. El sector primario se refiere a actividades de extracción directa de bienes de la naturaleza. Forma parte del sector primario la minería, la agricultura, la ganadería, silvicultura y la pesca. El sector secundario comprende las actividades que implican transformación de alimentos y materias primas a través de procesos productivos. En este sector se incluyen la siderurgia, las industrias mecánicas, químicas, textiles y producción de bienes de consumo. El sector terciario se dedica a ofrecer servicios a la sociedad, las personas $y$ las empresas.

Para explorar la asociación entre las variables de mortalidad y las de nivel socioeconómico y demográfico se llevó a cabo un análisis de correlación lineal simple utilizando gráficos de dispersión y el coeficiente de Pearson, considerándose como significativo un valor-p menor que 0,05 . Para evaluar el aporte conjunto de las variables socioeconómicas y demográficas en la mortalidad se ajustó un modelo de regresión lineal múltiple para cada región y sexo. El análisis de los datos, que incluye análisis de residuos, se realizó con el programa R 3.1.3.
Este estudio se llevó a cabo en cumplimiento con los principios éticos contenidos en la Declaración de Helsinki. No se requiere aprobación de comité ético-científico por el uso de datos secundarios (anónimos) de dominio público.

\section{Resultados}

En la Región Metropolitana, en hombres, 16 de las 52 comunas $(30,8 \%)$ presentan alto riesgo de mortalidad, en mujeres se observa $13,5 \%$ en alto riesgo. Además para ambos sexos, se observa mayor riesgo de mortalidad en las comunas de la Provincia de Santiago (en el centro de la Región), comunas netamente urbanas, y en comunas rurales de la periferia, se observan cifras menores. Se observan riesgos de mortalidad bajos en comunas del sector nororiente de esta región, las que son de mayor nivel socioeconómico. De las 54 comunas que conforman la Región del Bíobío, en hombres $44,4 \%$ y mujeres $16,7 \%$, presentan alto riesgo. En esta región se observa una agrupación con exceso de riesgo en comunas pertenecientes a los territorios de planificación del secano costero e interior, de vocación económica silvoagropecuaria (Figura 1$)^{18}$

Al analizar la correlación con variables socioeconómicas y demográficas en la Región Metropolitana, en hombres se presenta una correlación estadísticamente significativa con las variables ingresos $(r=-0,373)$, educación $(r=-0,248)$ (Figura 2$)$, porcentaje de población rural $(\mathrm{r}=-0,456)$, porcentaje de población perteneciente a pueblos originarios $(r=0,389)$ (Figura 3$)$, porcentaje de población económicamente inserta en el sector primario $(r=-0,447)$ (Figura 4$)$ y en el sector secundario $(r=0,553)$. En mujeres se encuentra una correlación estadísticamente significativa con las variables ingresos $(r=-0,599)$, educación $(\mathrm{r}=-0,481)$ (Figura 2), porcentaje de población perteneciente a pueblos originarios $(\mathrm{r}=0,539)$ (Figura 3 ) y porcentaje de población económicamente inserta en el sector secundario $(r=-0,646)$ (Figura 4).

En la Región del Bíobío, en hombres sólo se observó una correlación estadísticamente significativa con el porcentaje de población perteneciente a pueblos originarios $(\mathrm{r}=-0,301)$ (Figura 3 ) y en mujeres se encontró correlación con educación $(\mathrm{r}=0,297)$ (Figura 2), porcentaje de población 

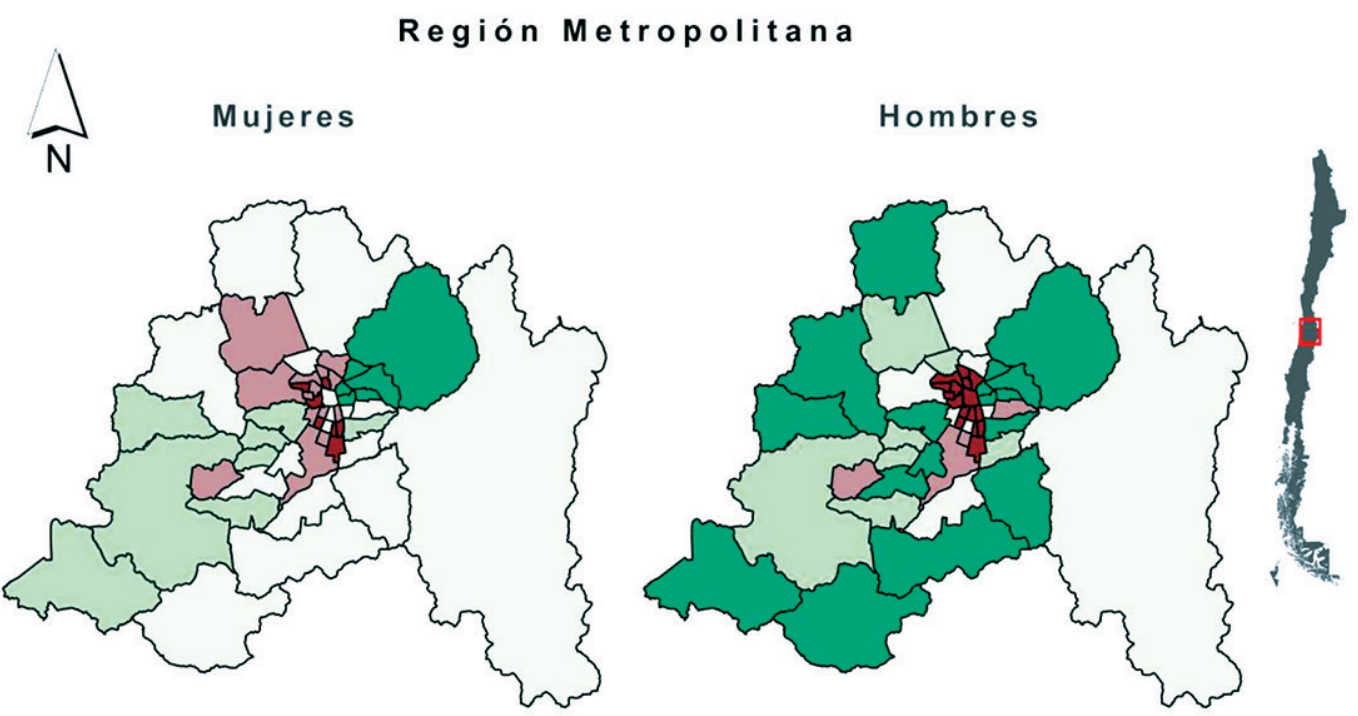

RMEs
$\square<70$
$\square 70-89$
$\square$ 90-109
$\square$ 110-129
口 >=130

Región del Bíobio
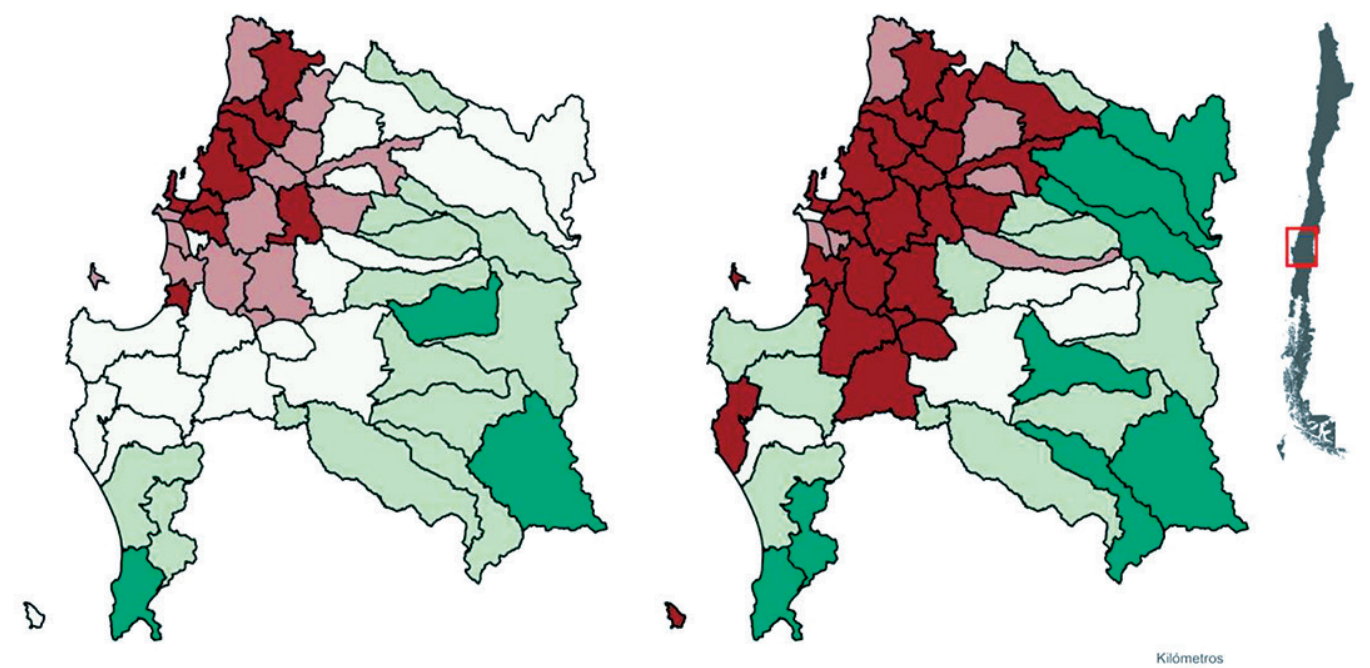

Figura 1. Razón de mortalidad estandarizada suavizada por cirrosis y otras enfermedades crónicas del hígado en hombres y mujeres de la Región Metropolitana y la Región del Bíobío 2001-2008. 


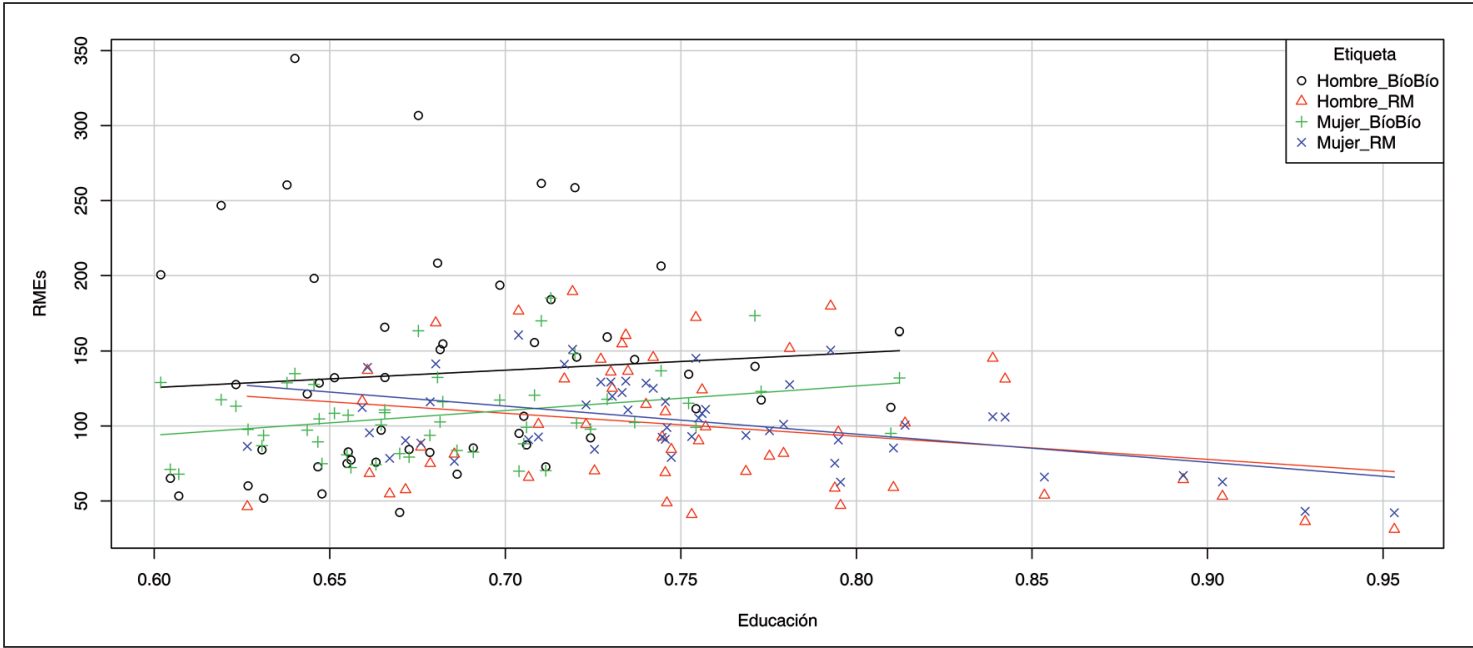

Figura 2. Razón de mortalidad estandarizada suavizada por cirrosis y otras enfermedades crónicas del hígado en hombres y mujeres de las regiones Metropolitana y Bíobío 2001-2008 y educación.

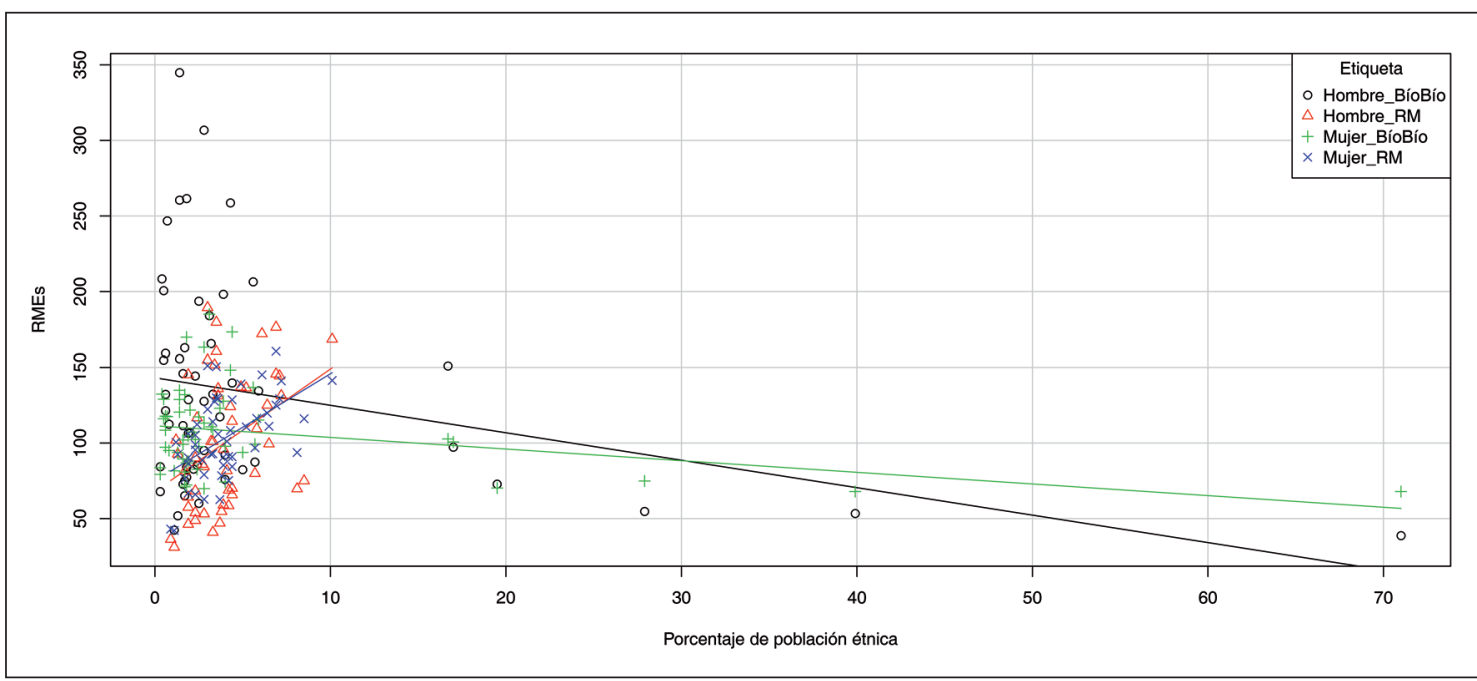

Figura 3. Razón de mortalidad estandarizada suavizada por cirrosis y otras enfermedades crónicas del hígado en hombres y mujeres de las regiones Metropolitana y Bíobío 2001-2008 y porcentaje de población perteneciente a pueblos originarios.

rural $(r=-0,327)$, porcentaje de población perteneciente a pueblos originarios $(\mathrm{r}=-0,321)$ (Figura 3), porcentaje de población económicamente inserta en el sector primario $(r=-0,272)$ (Figura 4) y en el sector terciario $(r=0,333)$ (Figura 5).

En los modelos multivariados de la Región Metropolitana en hombres y mujeres, las variables que mejor explican la RMEs son la educación y el porcentaje de población económicamente inserta en el sector primario, que en conjunto explican
$53,6 \%$ de la variabilidad total de la RMEs en hombres y $62,3 \%$ en mujeres. Comunas de menor educación tienen mayor riesgo de mortalidad por cirrosis, como también comunas donde hay menor proporción de trabajadores manuales.

En la Región del Bíobío en hombres se observa que el porcentaje de población perteneciente a pueblos originarios explica 9,1\% de la variabilidad total de la RMEs, en cambio en mujeres el porcentaje de población perteneciente a pueblos origina- 


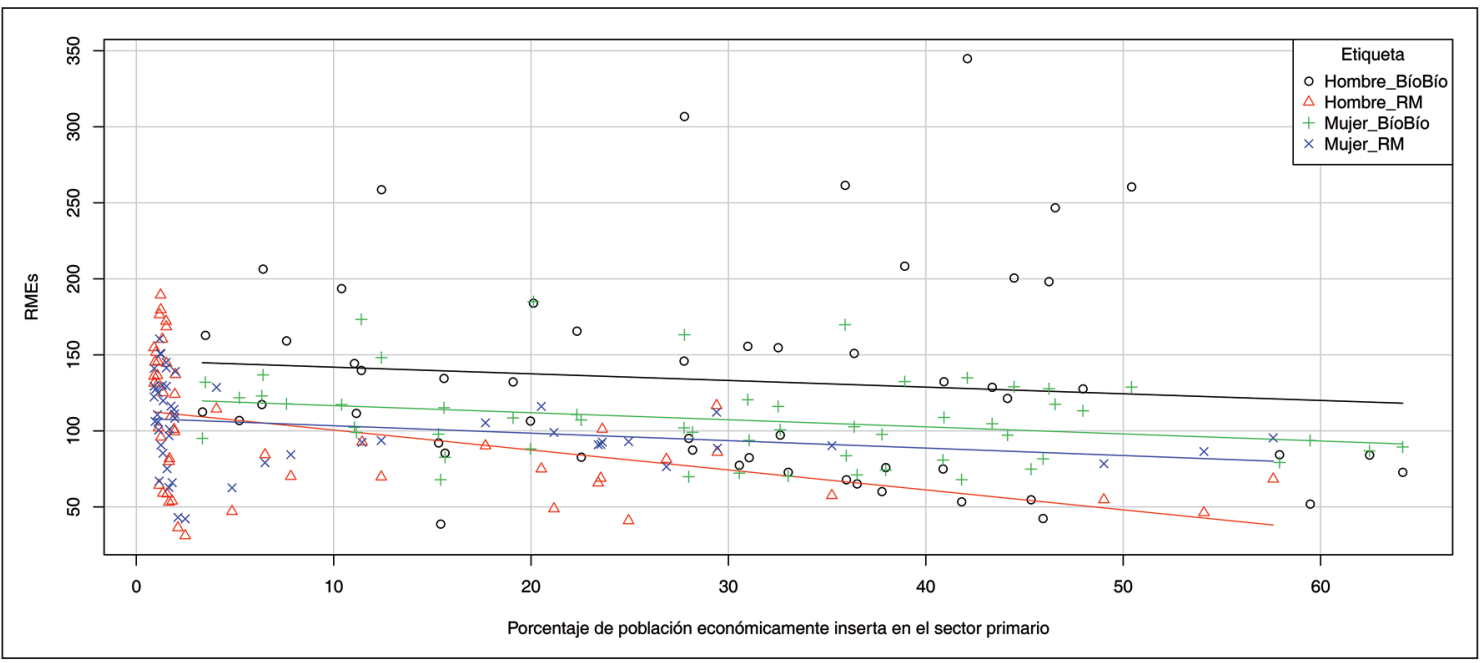

Figura 4. Razón de mortalidad estandarizada suavizada por cirrosis y otras enfermedades crónicas del hígado en hombres y mujeres de las regiones Metropolitana y Bíobío 2001-2008 y porcentaje de población económicamente inserta en sector primario.

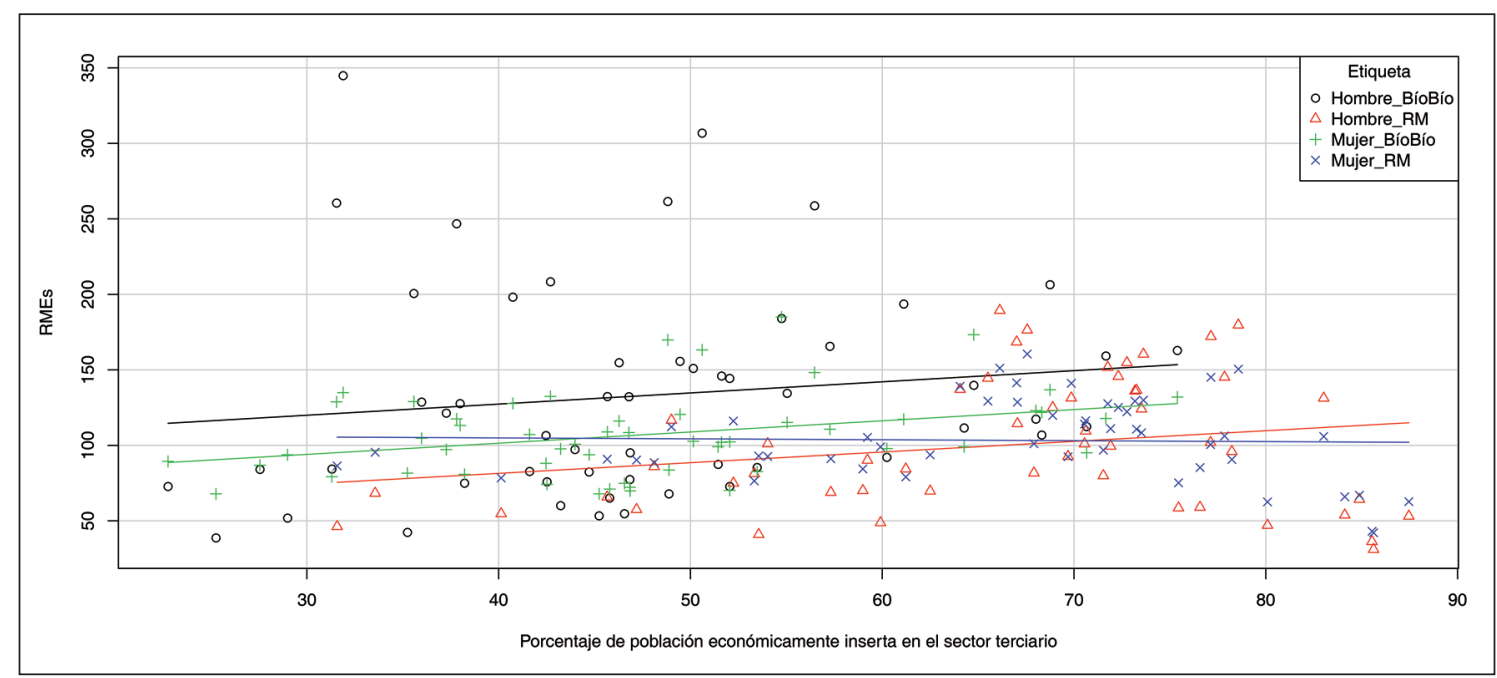

Figura 5. Razón de mortalidad estandarizada suavizada por cirrosis y otras enfermedades crónicas del hígado en hombres y mujeres de las regiones Metropolitana y Bíobío 2001-2008 y porcentaje de población económicamente inserta en sector terciario.

rios y el porcentaje de población económicamente inserta en el sector terciario explican 15,0\% de la variabilidad total de la RMEs. En hombres, comunas con menor porcentaje de población originaria tienen mayor riesgo de mortalidad por cirrosis. En el caso de las mujeres, además de las comunas con menor porcentaje de población originaria, el riesgo se asocia a comunas con mayor porcentaje de trabajadores/as asociados a servicios.

\section{Discusión}

En este estudio se analizó la asociación de diversos indicadores socioeconómicos y demográficos con el riesgo de mortalidad por cirrosis y otras enfermedades crónicas del hígado en las dos regiones más pobladas de Chile. Estos resultados son consistentes con los resultados observados en otros estudios a nivel nacional e internacional, que 
muestran que las áreas geográficas más deprivadas tienen peores indicadores de salud, incluyendo mortalidad cirrosis y por diversas causas ${ }^{19-21}$.

Los factores que estarían explicando el riesgo de mortalidad por cirrosis en la Región Metropolitana en hombres y mujeres son menor educación y menor porcentaje de población económicamente inserta en el sector primario, asociado a trabajos manuales. En cambio en la Región del Bíobío las variables que mejor explican el riesgo de mortalidad por cirrosis son el menor porcentaje de población perteneciente a pueblos originarios en hombres y mujeres; y el mayor porcentaje de población económicamente inserta en el sector terciario, asociado a servicios, solo en mujeres.

Este estudio muestra que existe diferentes realidades en estas dos regiones de Chile con respecto a los factores que influyen en el riesgo de morir por cirrosis hepática. Por otra parte, cabe hacer notar la similitud de los patrones geográficos y de las asociaciones entre hombres y mujeres, especialmente en la Región Metropolitana, similar a lo reportado por Emslie y Mitchell ${ }^{22}$. Destaca el caso de la Región del Bíobío, donde parte del riesgo de mortalidad por cirrosis en mujeres es además explicado por un mayor porcentaje de trabajos asociados a servicios.

Las comunas de la Región Metropolitana presentan una asociación esperable con los niveles de ingresos y educación: a menor nivel de ambas variables, mayor riesgo de mortalidad por cirrosis. Esta asociación se encuentra descrita para diversas enfermedades infecciosas y crónicas no-transmisibles, tanto en países desarrollados como en vías de desarrollo ${ }^{23-25}$ y se denomina como determinantes sociales de la salud. No obstante en el presente estudio la educación estaría jugando un rol más determinante en el exceso de mortalidad a nivel comunal. Aunque se debe considerar que la educación se encuentra asociada con el nivel de ingresos de las comunas. Por otra parte, en la Región del Bíobío se encuentran asociaciones opuestas con Ingresos y Educación en los modelo simples, la cual no fue estadísticamente significativa en los modelos multivariados.

En la Región Metropolitana se encontró una asociación positiva con el porcentaje de la población que pertenece a algún pueblo originario, mientras que en la región del Bíobío esta es negativa. Según un análisis de la población indígena chilena derivado de datos del censo del año
$2002^{26}$, las regiones Metropolitana y del Bíobío presentan una población indígena de 27,7\% y $7,8 \%$ respectivamente; ambas con alto porcentaje de población mapuche. Se describe en esta población cifras elevadas de analfabetismo, bajas tasas de participación económica y predominio de trabajadores asalariados. Esta aparente asociación positiva entre pertenencia a etnia mapuche y mayor vulnerabilidad socioeconómica podría explicar la correlación evidenciada en la Región Metropolitana. A pesar que en la cultura popular se asocia a la población mapuche con problemas de alcoholismo $^{27}$, no existe evidencia epidemiológica en Chile que muestre que las personas que pertenecen al pueblo mapuche presenten una mayor prevalencia de abuso y dependencia de alcohol que la población general ${ }^{28}$.

En cuanto a ruralidad, se encontró que el riesgo se asocia a comunas mayormente urbanas de la Región Metropolitana; resultado similar al encontrado en un estudio de Zaragoza, España ${ }^{21}$. Por otra parte, destaca la asociación negativa observada en la Región Metropolitana para la población económicamente inserta en el sector primario y positiva para la población económicamente inserta en el sector secundario. Según información del INE al año 2013 para la Región Metropolitana ${ }^{29}$, los ocupados en el sector primario representan un porcentaje menor del total de trabajadores $(3,4 \%)$. Como las actividades del sector primario en la Región Metropolitana se limitan a las comunas con ruralidad elevada, la asociación negativa de ambas con la mortalidad por cirrosis podría explicarse por factores comunes.

Dentro de las posibles limitaciones de este estudio se encuentra que a pesar de considerarse a Chile como un país con alta calidad en el reporte de causas de mortalidad $^{30}$, existen comunas con baja calidad de certificación médica ${ }^{31}$. Por otra parte, el sesgo ecológico, inherente a este tipo de estudio, dificulta establecer a priori la existencia de una asociación entre las variables a nivel individual. También se debe analizar con cautela que se trata de comparaciones entre datos agregados en el tiempo 2001-2008 y datos de años puntuales 2002 y 2006. Otra limitante es el número de comunas en cada región que dificulta el análisis desagregado por sectores urbano y rural.

Este estudio ha mostrado desigualdades en la mortalidad por cirrosis y otras enfermedades crónicas del hígado en hombres y mujeres resi- 
dentes de las regiones Metropolitana y Bíobío en el período 2001-2008. Esta aproximación al estudio de la relación entre variables socioeconómicas y demográficas y riesgo de mortalidad por cirrosis identificó mayor riesgo en las comunas de la Región Metropolitana pertenecientes a la provincia de Santiago, las cuales responderían a variables socioeconómicas y demográficas acorde a lo observado en la literatura internacional. En la Región del Bíobío destaca la agrupación de riesgo en las comunas del secano costero e interior. El estudio no logro identificar factores socioeconómicos y demográficos que explicasen la agregación de casos en estas comunas. Se hace necesario, bajo el entendido de la mortalidad de cirrosis como indicador de consumo crónico excesivo de alcohol, el explorar las realidades epidemiológicas en estas aglomeraciones identificadas para así establecer políticas de salud pública dirigidas hacia éstos.

\section{Referencias}

1. Tsochatzis EA, Bosch J, Burroughs AK. Liver cirrhosis. Lancet 2014; 383 (9930): 1749-61.

2. Mokdad AA, López AD, Shahraz S, Lozano R, Mokdad $\mathrm{AH}$, Stanaway, et al. Liver cirrhosis mortality in 187 countries between 1980 and 2010: a systematic analysis. BMC Med 2014; 12 (1): 145.

3. Ministerio de Salud, Departamento de Estadísticas e Información en Salud, DEIS. Bases de datos defunciones [Internet] Santiago, Chile: Ministerio de Salud, 2010. Disponible en: http://www.deis.cl/ [Consultado el 18 de octubre de 2016].

4. Alonso FT, Garmendia ML, de Aguirre M, Searle J. Análisis de la tendencia de la mortalidad por cirrosis hepática en Chile: Años 1990 a 2007. Rev Med Chile 2010; 138 (10): 1253-8.

5. Lefton HB, Rosa A, Cohen M. Diagnosis and epidemiology of cirrhosis. Med Clin N Am 2009; 93 (4): 787-99.

6. Mann RE, Smart RG, Govoni R. The epidemiology of alcoholic liver disease. Alcohol Res Heal 2003; 27 (3): 209-19.

7. World Health Organization, Department of Mental Health and Substance Dependence. International guide for monitoring alcohol consumption and related harm. Geneva(Switzerland): World Health Organization; 2000. 193 p. Report No.: WHO/MSD/MSB/00.4.

8. Ministerio del Interior y Seguridad Pública, Servicio Nacional para la prevención y rehabilitación del consumo de drogas y alcohol, SENDA. Décimo primer estudio nacional de drogas en población general [Internet]. Santiago, Chile: Observatorio Chileno de Drogas, 2015. 32 p. Disponible en: http://www.senda.gob.cl/wp-content/ uploads/2015/07/Informe-Ejecutivo-ENPG-2014.pdf [Consultado el 23 de febrero de 2016].

9. Ministerio de Salud, Subsecretaria de Salud Pública. Informe final: Estudio de carga de enfermedad y carga atribuible, Chile 2007 [Internet]. Santiago, Chile: Departamento de Epidemiología, 2008. 88 p. Disponible en: http://epi.minsal.cl/wp-content/uploads/2016/04/ Informe-final-carga_Enf_20071.pdf [Consultado el 20 de enero de 2016].

10. Castillo-Carniglia Á, Kaufman JS, Pino P. Alcohol-attributable mortality and years of potential life lost in Chile in 2009. Alcohol Alcohol 2013; 48 (6): 729-36.

11. Cutler D, Lleras-Muney A, Vogl, T. Socioeconomic status and health: Dimensions and mechanisms. In: Glied S, Smith PC, editors. The Oxford Handbook of Health Economics. New York: Oxford University Press; 2011. p. 124-63.

12. Icaza G, Núñez L, Torres-Avilés F, Díaz N, Villarroel JE, Soto A, et al. Atlas de mortalidad en Chile, 2001-2008. Talca, Chile: Editorial Universidad de Talca; 2013. 144 p.

13. Castillo-Carniglia Á, Kaufman JS, Pino P. Geographical distribution of alcohol-attributable mortality in Chile: A Bayesian spatial analysis. Addictive Behaviors 2015; 42: 207-15.

14. Beale L, Abellan JJ, Hodgson S, Jarup L. Methodologic issues and approaches to spatial epidemiology. Environ Health Perspect 2008; 116 (9): 1105-10.

15. Ministerio de Salud, Departamento de Epidemiología. Diagnósticos regionales en salud con enfoque de DDS. Bases de datos país nivel comunas 2009 [Internet] Santiago, Chile: Ministerio de Salud, 2012. Disponible en: http://epi.minsal.cl/estudios-y-encuestas-poblacionales/ diagnosticos-regionales [Consultado el 1 de septiembre de 2015].

16. Ministerio de Desarrollo Social. Encuesta CASEN. [Internet] Santiago, Chile: Ministerio de Desarrollo Social. 2006. Disponible en: http://observatorio.ministeriodesarrollosocial.gob.cl/casen/casen_obj.php [Consultado el 3 de mayo de 2015].

17. Programa de las Naciones Unidas para el Desarrollo, PNUD. Índice de desarrollo humano en Chile 19901998 [Internet]. Santiago, Chile: Programa de las Naciones Unidas para el Desarrollo. 1999. 39 p. Disponible en: http://desarrollohumano.cl/idh/download/todo.pdf [Consultado el 4 de mayo de 2015].

18. Comisión Nacional de Investigación Científica, CONICYT. Región del Bío Bío: Diagnóstico de las capacidades 
y oportunidades de desarrollo de la ciencia, la tecnología y la innovación. Santiago, Chile: Programa Regional de CONICYT, 2010. 96 p. Disponible en: http://www. conicyt.cl/regional/files/2013/06/Biobio.pdf [Consultado el 5 de mayo de 2015].

19. Santana P, Costa C, Marí-Dell'Olmo M, Gotsens M, Borrell C. Mortality, material deprivation and urbanization: exploring the social patterns of a metropolitan area. Int J for Equity in Health 2015; 14: 55.

20. Castillo-Carniglia A, Kaufman JS, Pino P. Small area associations between social context and alcohol-attributable mortality in a middle income country. Drug Alcohol Depend 2014; 137 (1): 129-36.

21. Aguilar I, Feja C, Compés ML, Rabanaque MJ, Esteban M, Alcalá T, et al. Desigualdades y mortalidad por cirrosis en varones (Zaragoza, 1996-2003). Gac Sanit 2011; 25 (2): 139-45.

22. Emslie C, Mitchell R. Are there gender differences in the geography of alcohol-related mortality in Scotland? An ecological study. BMC Public Health 2009; 9: 58.

23. Elo IT. Social class differentials in health and mortality: Patterns and explanations in comparative perspective. Annu Rev Sociol 2009; 35: 553-72.

24. Martikainen P, Mäkelä P, Koskinen S, Valkonen T. Income differences in mortality: a register based follow-up study of three million men and women. Int J Epidemiol 2001; 30 (6): 1397-405

25. Harrison L, Gardiner E. Do the rich really die young? Alcohol-related mortality and social class in Great Britain, 1988-94. Addiction 1999; 94 (12): 1871-80.
26. Instituto Nacional de Estadísticas y Ministerio de Planificación Nacional. Estadísticas sociales de los pueblos originarios en Chile-Censo 2002. [Internet]. Santiago, Chile: Instituto Nacional de Estadísticas, 2005. 200 p. Disponible en: http://www.ine.cl/canales/chile_estadistico/estadisticas_sociales_culturales/etnias/pdf/estadisticas_indigenas_2002_11_09_09.pdf [Consultado el 24 de mayo de 2015].

27. Godoy-Sepúlveda E, Hoces-Reyes K, Roa-Contreras C. Desmitificando la historia Mapuche: Contribuciones para la enseñanza de una nueva historia de Chile. [Seminario de titulación]. Santiago, Chile: Universidad de Santiago de Chile, Departamento de Historia; 2006. 152 p.

28. Vicente B, Kohn R, Rioseco P, Saldivia S, Torres S. Psychiatric disorders among the Mapuche in Chile. Int J Soc Psychiatry 2005; 51 (2): 119-27.

29. Instituto Nacional de Estadísticas [Internet]. Informe económico regional. Octubre-Diciembre de 2013. Santiago, Chile, Instituto Nacional de Estadísticas; 2014. 78 p. Disponible en: http://www.ine.cl/canales/chile_estadistico/territorio/iner/2013/IER_IV_trimestre_2013.pdf [Consultado el 24 de mayo de 2015].

30. Mahapatra P, Shibuya K, López AD, Coullare F, Notzon FC, Rao C, et al. Civil registration systems and vital statistics: successes and missed opportunities. Lancet 2007; 370 (9599): 1653-63.

31. Núñez L, Icaza G. Calidad de las estadísticas de mortalidad en Chile, 1997-2003. Rev Med Chile 2006; 134 (9): 1191-6. 